\title{
Utilização de gonadotrofina coriônica humana e cipionato de estradiol associado ao dispositivo de liberação controlada de drogas para sincronização de ovulação em cabras da raça Saanen
}

\author{
[Use of human chorionic gonadodrophin and estradiol cipionate plus controlled internal drug release for \\ synchronization of ovulation in Saanen goats] \\ V.V. Maffili ${ }^{1}$, C.A.A. Torres ${ }^{1}$, R.A.M. Pontes $^{2}$, J.D. Guimarães ${ }^{3}$, C.P. Prosperi ${ }^{4}$ \\ ${ }^{1}$ Departamento de Zootecnia - Universidade Federal de Viçosa - Viçosa, MG \\ ${ }^{2}$ Faculdade de Medicina Veterinária e Zootecnia - UNESP - Botucatu, SP \\ ${ }^{3}$ Departamento de Veterinária - Universidade Federal de Viçosa - Viçosa, MG \\ ${ }^{4}$ Departamento de Veterinária - Universidade Federal de Lavras - Lavras, MG
}

\begin{abstract}
RESUMO
Dezesseis cabras nulíparas da raça Saanen foram distribuídas em dois grupos de tratamentos (T1 e T2) para sincronização da ovulação. Inicialmente, ambos os tratamento consistiram na aplicação concomitante do dispositivo de liberação controlada de drogas $\left(C I D R-G^{\circledR}\right)$, de $5 \mathrm{mg}$ de dinoprost e de $1 \mathrm{mg}$ de cipionato de estradiol (CE) (dia 0). No quarto dia aplicaram-se 250UI de eCG e no quinto dia retirou-se o CIDR$\mathrm{G}^{\circledR}$. As cabras do $\mathrm{T} 1(\mathrm{n}=8)$ receberam $1 \mathrm{mg}$ de CE 24 horas depois da retirada do CIDR-G ${ }^{\circledR}$ e as do T2 $(\mathrm{n}=8)$ receberam $250 \mathrm{UI}$ de hCG 30 horas após. Sete cabras do T1 e oito do T2 entraram em estro depois da retirada do CIDR-G ${ }^{\circledR}$. Cabras que receberam hCG permaneceram em estro por $42,0 \pm 6,9$ horas e as que receberam $\mathrm{CE}$ por $45,0 \pm 5,5$ horas $(\mathrm{P}>0,05)$. As características ovulatórias não foram influenciadas pelos tratamentos. $\mathrm{O}$ intervalo da retirada do $\mathrm{CIDR}-\mathrm{G}^{\circledR}$ à ovulação para ambos os protocolos de sincronização da ovulação não diferiu $(\mathrm{P}>0,05)$ entre tratamentos. As ovulações promovidas pelo $\mathrm{CE}$ ocorreram em menor intervalo de tempo.
\end{abstract}

Palavras-chave: caprino, hCG, estrógeno

\begin{abstract}
Saanen nuliparous female goats were distributed in two experimental groups of eight animals each to synchronize the ovulation. On day zero, all animals were treated with controlled internal drug release $\left(C I D R-G^{\circledR}\right)$, dinoprost $(5 \mathrm{mg})$ and estradiol cipionate $(E C ; 1 \mathrm{mg})$. On day 4, all animals were treated with eCG (250IU), and on day 5 the CIDR-G ${ }^{\circledR}$ was removed. Group 1 (T1) received $1 m g$ of EC and Group 2 (T2) received 250IU of hCG, 24 and 30 hours after CIDR- $G^{\circledR}$ removal, respectively. Onset of estrus was observed in 7 and 8 goats of T1 and T2 groups, respectively. hCG treated goats remained in estrus for $42 \pm 6.9$ hours, whereas EC-treated goats remained in estrus for $45 \pm 5.5$ hours $(P>0.05)$. None of the studied ovulatory characteristics were affected by treatments. The intervals between $C I D R-G^{\circledR}$ removal and ovulation in T1 and T2 groups were similar. Both hCG and EC were equally efficient on inducing ovulation synchronically.
\end{abstract}

Keywords: caprine, $h C G$, estrogen

Recebido para publicação em 11 de dezembro de 2004

Recebido para publicação, após modificações, em 29 de junho de 2004

*Endereço para correspondência (mailing address)

Rua dos Passos, 664, Centro

36570-000 - Viçosa, MG

E-mail: vmaffili@hotmail.com 


\section{INTRODUÇÃO}

Os avanços no processo de criopreservação de sêmen caprino têm aumentado o interesse pelo uso da inseminação artificial (IA). Contudo, o sucesso dos programas de inseminação artificial está condicionado a fêmeas que ovulem durante um período de tempo previsível, principalmente se forem utilizados programas de inseminação artificial com tempo fixo (IATF). Não há consenso entre os pesquisadores sobre o momento ideal para a realização da IATF na espécie caprina. Baril et al. (1992) consideraram como o melhor momento 45 horas após a retirada do dispositivo liberador de progestágeno. Ritar et al. (1990) recomendaram a inseminação decorridas 55 horas da retirada da esponja. Corteel et al. (1984), citados por Ritar et al. (1990), sugeriram que a IA fosse feita de acordo com a raça, isto é, 45 e 49 horas após a retirada da esponja nas raças Alpina e Saanen, respectivamente. No Brasil, Machado e Simplício (2001) observaram melhores taxas de gestação em cabras inseminadas entre 44 a 50 horas após a retirada da esponja.

O $17 \beta$-estradiol (17 $\beta$-E) e o benzoato de estradiol (BE) são comumente utilizados para sincronização da emergência da onda folicular e ovulação em vacas tratadas com controlled internal drug release (CIDR-G) (Andrade et al., 2002; Colazo et al., 2003). O uso do cipionato de estradiol (CE), um éster do estradiol que apresenta níveis plasmáticos por períodos superiores a 100 horas, tem sido desestimulado por apresentar resultados, em termos de sincronização da emergência de onda, menos previsível do que fármacos de ação mais curta (Mapletoft et al., 2000), como o 17 $\beta$-E (Bo et al., 1995) e o BE (Cassia e Bo, 1995). Colazo et al. (2003), ao procederem análise comparativa do $\mathrm{CE}$ com o $17 \beta-\mathrm{E}$ e $\mathrm{BE}$, observaram que o $\mathrm{CE}$ foi menos eficaz em sincronizar a emergência da onda e ovulação, sem, no entanto, repercutir na taxa de gestação. Lopes et al. (2000) relataram o sucesso da utilização do CE em protocolos de IATF.

A gonadotrofina coriônica humana (hCG) tem sido utilizada principalmente em bovinos visando a indução de corpo lúteo acessório (Fonseca et al., 2001). Fonseca et al. (2002) demonstraram que, em caprinos, há aumento na concentração de progesterona. Tallam et al.
(2001) observaram que o aumento na concentração plasmática de progesterona não resultou em ganho na taxa de gestação. Rensis et al. (2002) não verificaram efeito sobre as características observadas quando são realizadas substituições da segunda dose do GnRH pela hCG e concluiram que o uso de outro hormônio está condicionado ao preço de aquisição e de outros benefícios advindos da escolha. Não há relatos na literatura consultada a respeito da utilização da hCG para sincronização da ovulação em caprinos. O objetivo do estudo foi o de comparar dois protocolos de sincronização de ovulação, tendo como hormônios indutores hCG e CE.

\section{MATERIAL E MÉTODOS}

O estudo foi realizado durante a estação reprodutiva, no mês de maio (outono), na granja Água Limpa, no município de Piau, Minas Gerais. A região, localizada a $21^{\circ} 35^{\prime} \mathrm{S}$ e $43^{\circ} 15^{\prime} \mathrm{W}$ e a 435 metros de altitude, apresenta clima do tipo Cwa, segundo a classificação de Koppen.

Dezesseis fêmeas da raça Saanen, com peso médio de $36,00 \pm 3,76 \mathrm{~kg}$ e escore corporal médio de $3,31 \pm 0,31$, foram divididas, aleatoriamente, em dois grupos de oito (T1 e T2) animais. No $\mathrm{T}$ 1, a sincronização da ovulação consistiu na inserção do CIDR-G ${ }^{\circledR 1}$ (dia 0$)$ associado à aplicação intra-muscular de $5 \mathrm{mg}$ de dinoprost ${ }^{2}$ (análogo sintético da $\mathrm{PGF}_{2 \alpha}$ ) e de $1 \mathrm{mg}$ de $\mathrm{CE}^{3}$. No dia quatro foi aplicado 250UI de gonadotrofina coriônica equina ${ }^{4}$ (eCG), via intramuscular. A retirada do CIDR-G ocorreu no quinto dia, e 24 horas foi aplicado $1 \mathrm{mg}$ de $\mathrm{CE}$ (Billings e Katz, 1999). No T2 empregou-se o mesmo protocolo de sincronização, substituindose o CE por $250 \mathrm{UI} \mathrm{hCG}^{5}$, aplicados 30 horas após a retirada do CIDR-G ${ }^{\circledR}$.

Os animais, mantidos em confinamento, receberam capim-napier (Panicum maximum) picado, concentrado comercial $^{6}$, sal mineral e água à vontade.

\footnotetext{
${ }^{1}$ Eazi-Breed CIDR-G ${ }^{\circledR}$, Pharmacia Saúde Animal do Brasil Ltda

${ }^{2}$ Lutalyse $^{\circledR}$ - Pharmacia Saúde Animal do Brasil Ltda

${ }^{3} \mathrm{ECP}^{\circledR}$, Pharmacia Saúde Animal do Brasil Ltda

${ }^{4}$ Novormon ${ }^{\circledR} 5000$, Tecnopec Ltda

${ }^{5}$ Vetecor ${ }^{\circledR}$, Laboratório Calier do Brasil Ltda

${ }^{6}$ Advanced $^{\circledR}$ - Agroceres
} 
A cada seis horas, contando-se imediatamente após a retirada dos dispositivos intravaginais, as fêmeas passaram a ser monitoradas no intuito de detectar o início do estro. Os sinais de estro observados foram: procura pelo macho, inquietação, vocalização, micção freqüente, batimento de cauda, contração, hiperemia e edema de vulva, descarga de muco vaginal e imobilidade à monta, característica esta considerada como início do estro.

Em seguida à aplicação dos hormônios para sincronização - dia zero - iniciou-se a avaliação da dinâmica folicular ovariana utilizando-se, para isso, ultra-som ${ }^{7}$, acoplado a uma probe de $5 \mathrm{MHz}$ por via trans-retal (Menchaca e Rubianes, 2001). As avaliações foram realizadas uma vez ao dia, sempre às 18 horas. Após detectado o início do estro, a avaliação passou a ser realizada a cada seis horas e até 12 horas após a ovulação. Os procedimentos adotados para localização dos ovários seguiram os preconizados por Ginther e Kot (1994), tendo-se o cuidado de anotar em fichas individuais o diâmetro, a posição e as características das estruturas ovarianas. $\mathrm{O}$ acompanhamento possibilitou ainda definir a emergência da onda folicular como sendo a emergência de um pool de pequenos folículos com diâmetro médio entre 3,0 e 4,0mm que, por sua vez, deram origem a um ou mais folículos com diâmetros maior ou igual a $5,0 \mathrm{~mm}$. $\mathrm{O}$ dia da emergência da onda coincidiu com aquele em que o folículo apresentava $3,0 \mathrm{~mm}$ de diâmetro, aumentado a seguir para valores iguais ou superiores a 4,0mm (Rubianes et al., 2003). O diâmetro folicular foi obtido pela média da maior distância $(\mathrm{mm})$ entre dois pontos da cavidade antral dos folículos a partir de $2,5 \mathrm{~mm}$. A taxa de crescimento do folículo ovulatório $(\mathrm{mm} / \mathrm{dia})$ foi obtida por meio da diferença dos diâmetros final e inicial, dividido pelo número de horas e multiplicado por 24. A ovulação foi calculada pela média entre o horário da última observação do folículo ovulatório e o horário assinalado quando se detectou a ovulação.

Amostras de sangue foram coletadas por punção da jugular, às 24 e 48 horas após a retirada do CIDR-G ${ }^{\circledR}$, em tubos com vácuo e anticoagulante (EDTA) e acondicionados em banho de gelo. O sangue foi centrifugado a $3000 \mathrm{rpm}$ por 15 minutos e o plasma separado e estocado a $-20^{\circ} \mathrm{C}$ até a análise. Para determinar a concentração plasmática de progesterona utilizou-se a técnica de radioimunoensaio (RIA) de fase sólida (sensibilidade de $0,03 \mathrm{ng} / \mathrm{ml}$ ), optando-se pelo uso de kits comerciais e seguindo as recomendações do fabricante.

$\mathrm{Na}$ análise estatística foi utilizado o programa SAEG 8.0 (Sistema..., 1997). Para verificar a normalidade e a homogeinidade das variáveis estudadas foram empregados os testes de Lilliefors e Cochran e Barttlet, respectivamente. Empregaram-se a análise de variância e teste de médias de Student Newman Keuls. As variáveis que não apresentavam normalidade ou homogeinidade mesmo após a transformação logaritímica foram submetidas à análise não paramétrica e as médias comparadas pelo teste de Wilcoxon.

\section{RESULTADOS E DISCUSSÃO}

Das 16 cabras submetidas ao protocolo de ovulação, somente uma cabra no T1 $(6,2 \%)$ não apresentou sinais de estro (Tab. 1). As concentrações de progesterona mostraram-se acima de $1 \mathrm{ng} / \mathrm{ml} 24$ e 48 horas depois da retirada do CIDR-G ${ }^{\circledR}$. As demais cabras apresentaram valores abaixo de $1 \mathrm{ng} / \mathrm{ml} 24$ e 48 horas depois da retirada do CIDR-G. As cabras de T1 apresentaram sinais mais nítidos de estro, maior descarga vulvar, exarcebado comportamento homossexual e vulva mais edemaciada do que as cabras do T2. Relatos semelhantes foram observados em bovinos (Martinez et al., 2002).

Tabela 1. Porcentagem de animais em estro (AE), intervalo retirada do CIDR-G e início do estro (IE), duração do estro (DE) e intervalo retirada do CIDR-G e fim do estro (IF) em cabras submetidas à sincronização de ovulação com cipionato de estradiol (T1) ou gonadotrofina coriônica humana (T2) (média $\pm \mathrm{DP})$

\begin{tabular}{|c|c|c|c|}
\hline & T1 (E2) & T2 (hCG) & Total (n) \\
\hline $\mathrm{AE}$ & $87,5 \%(7 / 8)$ & $100 \%(8 / 8)$ & $93,8 \%(15 / 16)$ \\
\hline IE (hora) & $24,0 \pm 6,0 \mathrm{a}$ & $20,2 \pm 5,5 \mathrm{a}$ & $22,0 \pm 5,8$ \\
\hline DE (hora) & $42,0 \pm 6,9 \mathrm{a}$ & $45,0 \pm 5$ & $43,6 \pm 6,2$ \\
\hline IF (hora) & $66,0 \pm 6,9 \mathrm{~A}$ & $65,2 \pm 3,8 \mathrm{~A}$ & $65,6 \pm 5,3$ \\
\hline \multicolumn{4}{|c|}{$\begin{array}{l}\text { Médias seguidas por letras distintas minúsculas na linha } \\
\text { diferem entre si pelo teste } \mathrm{SNK}(\mathrm{P}<0,05) \text {. } \\
\text { Médias seguidas por letras distintas maiúsculas na linhe } \\
\text { diferem entre si pelo teste Wilcoxon }(\mathrm{P}<0,05) \text {. } \\
\mathrm{DP}=\text { desvio-padrão }\end{array}$} \\
\hline
\end{tabular}

${ }^{7}$ Aloka, modelo SSD-500 
Houve similaridade na duração do estro entre os grupos $(\mathrm{P}>0,05)$, e o intervalo retirada do CIDR$\mathrm{G}$ e final do estro não diferiu entre $\mathrm{T} 1(66,0 \pm 6,9)$ e T2 $(65,2 \pm 3,8)(\mathrm{P}>0,05)$ (Tab. 1).

O esquema proposto para promover regressão folicular mostrou-se eficiente, pois todos os folículos apresentaram-se em processo de atresia durante a permanência do dispositivo, havendo a emergência de nova onda no dia quatro. O intervalo entre a retirada do CIDR-G e o início do estro mostrou-se semelhante em ambos os tratamentos $(\mathrm{P}>0,05)$, com média geral de $22,0 \pm 5,8$.

$\mathrm{A}$ aplicação do $\mathrm{CE}$ ou hCG não influenciou o número de ovulações $(\mathrm{P}>0,05)$ (Tab. 2). Das 27 ovulações, 14 ocorreram nas cabras do T1 $(2,0 \pm 0,80)$ e 13 nas do T2 $(1,6 \pm 0,74)$. Assim como no número de ovulações, os protocolos de sincronização empregados não influenciaram o diâmetro médio dos folículos ovulatórios $(\mathrm{P}>0,05)$. As taxas de crescimento médio dos folículos ovulatórios foram de 2,2 $\pm 0,96 \mathrm{em}$ cabras que receberam $\mathrm{CE}$, e de 2,0 0 , 0,99 nas que receberam hCG, demonstrando que as taxas não diferiram entre os grupos $(\mathrm{P}>0,05)$.

Os dados sobre os diferentes intervalos do momento de ovulação e entrada em estro, final do estro, retirada do CIDR-G e aplicação do CE ou da hCG são mostrados na Tab. 2. O intervalo médio do início do estro e ovulação foi de $34,4 \pm 6,6$ para ambos os tratamentos, não se observando diferença entre eles $(\mathrm{P}>0,05)$. Das 15 cabras que entraram em estro, uma ovulou após o término do estro, duas ovularam no momento final do estro e as demais antes do término do estro. As cabras do T1 e T2 ovularam em média $10,3 \pm 4,5$ e $8,2 \pm 4,5$ horas antes do término do estro, respectivamente $(\mathrm{P}>0,05)$. A diferença observada entre o intervalo da aplicação da hCG ou $\mathrm{CE}$ e o momento da ovulação deveu-se basicamente à diferença entre os momentos de aplicação dos hormônios.

Na Fig. 1 encontram-se o número de cabras submetidas aos tratamentos segundo o intervalo retirada do CIDR-G e o momento de ovulação. O $\mathrm{CE}$ e a hCG foram eficientes em induzir a ovulação e promoverem ovulações em um período de tempo uniforme.
Tabela 2. Número de ovulações por estro (NO), intervalo do início do estro e momento de ovulação (IEMO), intervalo do final do estro e momento de ovulação (FEMO), intervalo da retirada do CIDR-G estro e momento de ovulação (IRD), diâmetro médio dos folículos ovulatórios (DMFO) e taxa de crescimento médio dos folículos ovulatórios (TCFO) em cabras submetidas à sincronização de ovulação com cipionato de estradiol (T1) ou gonadotrofina coriônica humana (T2) (média \pm DP)

\begin{tabular}{lccc}
\hline & T1 & T2 & Média geral (n) \\
\hline NO & $2,0 \pm 0,80 \mathrm{a}$ & $1,6 \pm 0,74 \mathrm{a}$ & $1,8 \pm 0,77$ \\
IEMO (hora) & $31,7 \pm 5,7 \mathrm{a}$ & $36,7 \pm 6,8 \mathrm{a}$ & $34,4 \pm 6,6$ \\
FEMO (hora) & $-10,3 \pm 4,5 \mathrm{a}$ & $-8,2 \pm 4,5 \mathrm{a}$ & $-9,2 \pm 4,5$ \\
IRD (hora) & $55,7 \pm 2,9 \mathrm{~A}$ & $57,0 \pm 5,5 \mathrm{~A}$ & $56,4 \pm 4,4$ \\
DMFO (mm) & $7,5 \pm 1,2 \mathrm{a}$ & $8,3 \pm 1,7 \mathrm{a}$ & $7,9 \pm 1,5$ \\
TCFO (mm/dia) & $2,2 \pm 0,96 \mathrm{a}$ & $2,0 \pm 0,99 \mathrm{a}$ & $2,1 \pm 0,97$ \\
\hline Médias seguidas por letras distintas minúsculas na linha diferem \\
entre si pelo teste SNK (P $<0,05)$. \\
Médias seguidas por letras distintas maiúsculas na linha diferem \\
entre si pelo teste Wilcoxon (P<0,05). \\
DP= desvio-padrão.
\end{tabular}

A hCG, por apresentar efeito fisiológico semelhante ao hormônio luteinizante (LH), vem sendo utilizada como hormônio indutor de ovulação em bovinos, pois sua utilização promoveria a maturação e ovulação de folículos de forma sincrônica por apresentar longa meiavida, em torno de 10 horas (Rensis et al., 2002). Neste estudo, observou-se que as ovulações promovidas pela hCG apresentaram-se mais variáveis do que as observadas com o estradiol. Os resultados deste estudo desencorajam a utilização da hCG em protocolos de sincronização de ovulação em caprinos. Embora tenha induzido $100 \%$ de ovulações, elas ocorreram num grande intervalo de tempo, entre 48 e 66 horas após a retirada do CIDR-G, o que inviabilizou a sua utilização em programas de IATF. Por ser um hormônio glicoprotéico de origem humana, seu uso rotineiro pode levar à formação de anticorpos, o que culminaria na redução de atividade biológica. Outro fator a ser considerado é o elevado custo de aquisição desse hormônio.

Em bovinos o $\mathrm{CE}$ apresenta resposta mais variável em termos de sincronia de onda e ovulação quando comparado ao benzoato de estradiol e o 17 $\beta$-estradiol (Colazo et al., 2003). No presente estudo tais efeitos não foram observados, pois em todas as cabras houve regressão folicular e as ovulações ocorreram dentro de um intervalo curto (entre 54 e 60 horas após a retirada do dispositivo). 


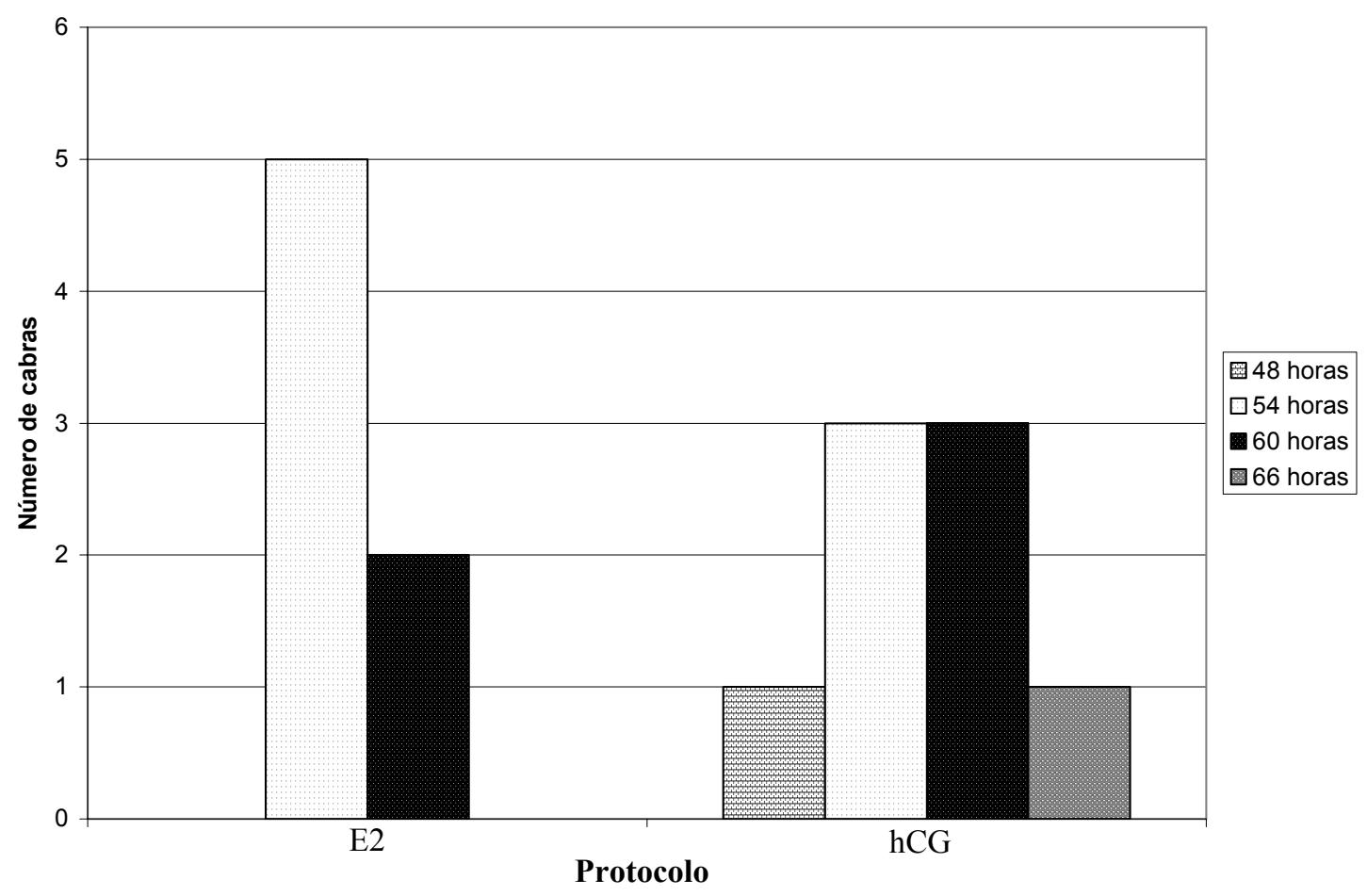

Figura 1. Número de cabras submetidas à sincronização de ovulação com cipionato de estradiol (CE) ou gonadotrofina capiônica humana ( $\mathrm{hCG}$ ) de acordo com o intervalo retirada do CIDR-G e momento de ovulação.

Os resultados deste estudo reforçam a tese do efeito bifásico da aplicação do estradiol. Assim, em estudos pertinentes ao controle da secreção de gonadotrofinas, tem sido demonstrado que em ovelhas (Caraty et al., 1995) e em bovinos (Bo et al., 2000), a administração exógena de estradiol induz à liberação pré-ovulatória de LH em ovinos, decorridas aproximadamente 11 horas (Meikle et al., 2001) e bovinos (Bo et al., 2000). Karsch et al. (1997) relataram que a liberação de LH induzida pelo estradiol está associada a eventos neuroendócrinos que culminaram na liberação de $\mathrm{GnRH}$, atrelado ao aumento da sensibilidade da hipófise ao GnRH (Clarke et al., 1988), por aumentar o número de receptores hipofisário para esse hormônio. Butler et al. (1983) descreveram o efeito bifásico da administração do estradiol, ou seja, quando administrado na ausência de progesterona, ocorre elevação na concentração de LH porém, quando associado à progesterona, promove regressão do folículo dominante e emergência sincrônica de uma nova onda. Neste estudo observou-se efeito bifásico da administração, o que significa dizer que na presença do CIDR-G houve regressão folicular. Entretanto, na ausência de concentração lútea de progesterona, o estradiol induziu à ovulação em todas as cabras que responderam ao tratamento de forma sincrônica.

O intervalo entre a retirada do CIDR-G e o momento de ovulação não diferiu entre os dois protocolos, apresentando intervalo médio de 56 horas. Este resultado implica que a inseminação deve ser realizada por volta de 48 horas após a retirada do dispositivo, e confirma os resultados de Corteel et al. (1984), citados por Ritar et al. (1990), para a raça Saanen. Diferem, todavia, dos preconizados por Ritar et al. (1990), quando empregaram esponjas impregnadas com $60 \mathrm{mg}$ de acetato de medroxiprogesterona. Machado e Simplício (2001) observaram que as melhores taxas de gestação foram obtidas quando as cabras foram inseminadas entre 44 e 48 horas após a retirada do dispositivo. Contudo, deve ser ressaltado que esses autores não utilizaram hormônios indutores de ovulação. 


\section{CONCLUSÕES}

O uso do cipionato de estradiol e progesterona do CIDR-G foi efetivo em sincronizar a emergência de nova onda de crescimento folicular. $O$ esquema proposto para indução de ovulação mostrou-se eficiente, levando a ovulações de forma sincrônica. Com base no protocolo proposto, recomenda-se inseminação em tempo fixo, 48 horas após a retirada do dispositivo.

\section{REFERÊNCIAS BIBLIOGRÁFICAS}

ANDRADE， J.C.O.; OLIVEIRA， M.A.L.; LIMA, P.F. et al. Use of steroid hormone treatments prior to superovulation in Nelore donors. Anim. Reprod. Sci., v.69, p.9-14, 2002.

BARIL, G.; REMY, B.; VALLET, J.C. et al. Effect of repeated use of progestagen-PMSG treatment for estrus control in dairy goats out of breeding season. Reprod. Dom. Anim., v.27, p.161-168, 1992

BILLINGS, H.J.; KATZ, L.S. Facilitation of sexual behavior in French-Alpine goats treated with intravaginal progesterone-releasing devices and estradiol during the breeding and nonbreeding seasons. J. Anim. Sci., v.77, p.20732078, 1999.

BO, G.A.; ADAMSN, G.P.; MAPLETOFT, R.J. Exogenous control of follicular wage emergence in cattle. Theriogenology, v.43, 31-40, 1995.

BO, G.A.; BERGFELT, D.R.; BROGLIATTI, G.M. et al. Local versus systemic effects of exogenous estradiol-17 $\beta$ on ovarian follicular dynamics in heifers with progestogen implants. Anim. Reprod. Sci., v.59, p.141-157, 2000.

BUTLER, W.R.; KATZ, L.S.; ARRIOLA, J. et al. On the negative feed-back regulation of gonadotropins in castrate and intact cattle with comparison of two FSH radioimmunoassays. $J$. Anim. Sci., v.56, p.919-919, 1983.

CARATY, A.; EVANS, N.P.; FABRE-NYS, C.J. et al. The preovulatory gonadotrophinreleasing hormone surge: a neuroendocrine signal for ovulation. J. Reprod. Fertil., v.49, p.245-255, 1995.

CASSIA, M.; BO, G.A. Follicle wave emergence following treatment of CIDR-G-B implanted beef heifers with estradiol benzoate and progesterone. Theriogenology, v.49, p.341, 1995. Abstract.

CLARKE, I.J.; CUMMINS, J.T.; CROWDER, ME.E. et al. Pituitary receptors for gonadotrophin-release hormone in relation to changes in pituitary and plasma gonadotrophins in ovariectomized hypothalamo/pituitarydisconnected ewes. II. A marked rise in receptor number during the acute feedback effects of estradiol. Biol. Reprod., v.39, p.349-354, 1988.

COLAZO, M.G.; KASTELIC, J.P.; MAPLETOFT, R.J. Effects of estradiol cypionate (ECP) on ovarian follicular dynamics, synchrony of ovulation, and fertility in CIDR-Gbased, fixed-time AI programs in beef heifers. Theriogenology, v.60, p.855-865, 2003.

FONSECA, J.F. Controle e perfil hormonal do ciclo estral e performance reprodutiva de cabras Alpina e Saanen. 2002. 107f. Tese (Doutorado em Zootecnia) - Departamento de Zootecnia, Universidade Federal de Viçosa, Viçosa, MG.

FONSECA, J.F.; SILVA FILHO, J.M.; PINTO NETO, A. et al. Taxas de gestação em novilhas receptoras submetidas à administração de rbST, GnRH ou hCG no quinto dia do ciclo estral. Arq. Bras. Med. Vet. Zootec., v.53, p.459-464, 2001

GINTHER, O.J.; KOT, K. Follicular dynamics during the ovulatory season in goats. Theriogenology, v.42, p.987-1001, 1994.

KARSCH, F.J.; BOWEN, J.M.; CARATY, A. et al. Gonadotrophin-releasing hormone requirements for ovulation. Biol. Reprod., v.56, p.303-309, 1997.

LOPES, F.L.; ARNOLD, D.R.; WILLIAMS, J. et al. Use of estradiol cypionate for timed insemation. J. Dairy Sci., v.83, p.216, 2000. (Abstract).

MACHADO, R.; SIMPLÍCIO, A.A. Avaliação de programas hormonais para a indução e sincronização do estro em caprinos. Pesq. Agrop. Bras., v.36, p.171-178, 2001.

MAPLETOFT, R.J.; BO, G.A.; ADAMS, G.P. Avanços na manipulação do ciclo estral de doadoras e receptoras nos programas de transferência de embriões em bovinos. In: REUNIÃO ANUAL DA SOCIEDADE BRASILEIRA DE TECNOLOGIA DE 
EMBRIÕES, 15., 2000, Rio Quente. Anais... Rio Quente: SBTE, 2000. p.24-51.

MARTÍNEZ, M.F.; KASTELIC, J.P.; ADAMS, G.P. et al. The use of progestins in regimens for fixed-time artificial insemination in beef cattle. Theriogenology, v.57, p.1049-1059, 2002.

MEIKLE, A.; FORSBERG, M.; GARÓFALO, E.G. et al. Circulating gonadotrophins and follicular dynamics in anestrous ewes after treatment with estradiol 17- $\beta$. Anim. Reprod. Sci., v.67, p.79-90, 2001.

MENCHACA, A.; RUBIANES, E. Effect of high progesterone concentrations during the early luteal phase on the lenght of the ovulatory cycle of goats. Anim. Reprod. Sci., v.68, p.69-76, 2001.

RENSIS, F.; MARCONI, P.; CAPELLI, T. et al. Fertility in postpartum dairy cows in winter or summer following estrus synchronization and fixed time AI after the induction of an LH surge with GnRH or hCG. Theriogenology, v.58, p.1675-1687, 2002.
RITAR, A.J.; O’MAY, P.J.; BALL, P.D. Artificial insemination of Cashmere goats: effects on fertility and fecundity of intravaginal treatment, method and time of insemination, semen freezing process, number of motile spermatozoa and age of females. Reprod. Fertil. Develop., v.2, p.377-384, 1990.

RUBIANES, E; MENCHACA, A.; CARBAJAL, B. Response of the 1-5 day-aged ovine corpus luteum to prostaglandin F2 $\alpha$. Anim. Reprod. Sci., v.78, p.47-55, 2003.

SISTEMA de análises estatísticas e genéticas SAEG. Viçosa: UFV, 1997. 59p.

TALLAM, S.K.; KERBLER, T.L.; LESLIE, K.E. et al. Reproductive performance of postpartum dairy cows under a highly intervenient breeding program involving timed insemination and combinations of $\mathrm{GnRH}$, prostaglandin $\mathrm{F}_{2 \alpha}$ and human chorionic gonadotrofin. Theriogenology, v.56, p.91-104, 2001. 